\title{
Absence of Phosphatidylglycerol (PG) in Respiratory Distress Syndrome in the Newborn
}

\author{
Study of the Minor Surfactant Phospholipids in Newborns
}

\author{
MIKKO HALLMAN, BERNARD H. FELDMAN, ELSA KIRKPATRICK, AND LOUIS GLUCK(40) \\ Department of Pediatrics, School of Medicine, University of California, San Diego, La Jolla, California, USA
}

\begin{abstract}
Summary
Phosphatidylglycerol (PG) was absent from lung effuent in $\mathbf{4 1}$ infants with respiratory distress syndrome of the newborn (RDS), whereas effluent from healthy control subjects of similar gestational age contained this phospholipid (4.9 $\pm 2.4 \%$ of lipidphosphorus (P), $n=32$ ). Control infants of 28 weeks of gestation or less with various respiratory disturbances other than RDS also had low PG (0.2 $\pm 0.2 \%$ of lipid-P, $n=5)$. In RDS surfactant complex often could be isolated from the airways using differential and density gradient centrifugation. The material thus obtained had prominent phosphatidylinositol (PI) (13.6 $\pm 2.8 \%$ of lipid-P, $n=6$ ), but no PG. Of those 18 infants who had such surfactant even in the early stages of RDS, 13 were 35 weeks of gestation or more, 3 were offspring of diabetic mothers, and 2 had severe perinatal asphyxia. In healthy control subjects PG sometimes appeared first within an hour of birth, but in RDS PG did not appear until recovery from RDS.

In RDS type II (transient tachypnea of the newborn) PG in lung effuent also was abnormally low $(1.3 \pm 0.6 \%$ of lipid-P, $n$ $=5)$ and PI was correspondingly prominent $(9.7 \pm 3.6 \%$ of lipid-P, $n=5$ ), indicating immaturity of surfactant similar to RDS.

Surfactant with PG and PI has superior surface-active properties compared to that containing PI, but no PG. Surfactant without PG does not seem to stabilize the alveoli of the newborn as well as does surfactant with PG. The failure of PG appearance following birth therefore may precipitate RDS, especially beyond 35 weeks of gestation.
\end{abstract}

\section{Speculation}

Pre- and postnatal monitoring of the acidic phospholipids, PG and PI, in lung effluent is useful in diagnosis and follow-up of RDS as well as in evaluation of various therapies.

Idiopathic RDS of the newborn is a disease of progressive atelectasis of the lungs with consequent hypoxia and characteristic pathology $(9,12)$. Several lines of evidence imply that in RDS lung surfactant is deficient, thus causing the tendency for atelectasis of the terminal airways. Babies with RDS have decreased compliance of their lungs (change in volume per unit change in pressure) $(2,25)$. Physiochemical and biochemical analyses indicate that the material recovered from the airways is deficient in surfactant $(3,15)$. Finally, various methods for distending the airways by artificial ventilation improve the otherwise grave prognosis $(6,10,18)$.

Surface activity and lung stability follow a developmental time table, first evident in late fetal life $(12,14)$. This development apparently can be accelerated by drugs such as corticosteroids
$(7,30)$. Betamethasone administered to mothers in premature labor decreases both the incidence and mortality of RDS (28). The analysis of surfactant in amniotic fluid by means of lecithin to sphingomyelin $(\mathrm{L} / \mathrm{S})$ ratio has proven to be a reliable way to predict the risk of RDS $(11,13)$. Therefore, the deficiency in surfactant seems to be the primary factor precipitating RDS.

Surfactant, especially that of the neonate, has a rapid turnover, and is removed by lymphatics and by ciliary action, necessitating continuous replenishment. By far the major fraction of surfactant consists of lecithin, predominantly with saturated fatty acids (disaturated lecithin). Its de novo biosynthesis in the lung principally takes place via the CDP-choline incorporation pathway $(8,17,35)$. The methylation of phosphatidylethanolamine to lecithin also may be active but of relatively minor significance in premature babies $(8,15)$.

Besides lecithin the surfactant complex contains other highly characteristic components. In the adult the second major phospholipid is, uniquely PG $(20,31,33)$, whereas, in contrast to the earlier reports, phosphatidyl dimethylethanolamine (PDME) is absent. In the fetal rabbit PG is absent, appearing first at birth. Another surfactant phospholipid, PI, is present in the fetus but then decreases concomitantly with the increase in PG (21).

The lung effluent of the newborn is a source of surfactant; its analysis enables the monitoring of the secretory function of the alveolar epithelial cells (15). In the present investigation pulmonary surfactant from the newborn was measured. In infants with RDS, as compared to healthy control subjects, a consistent specific difference was found in the acidic phospholipids of lung effluent but not always in lecithin.

\section{MATERIALS AND METHODS}

The present material included 302 samples of lung effluent obtained from 112 newborns at University Hospital, San Diego. The healthy patients as well as $36 \%$ of the sick patients were born at the same hospital; the rest came from surrounding units within 200 miles. The infants included in this study were of appropriate sizes for their gestations (between 10th and 90th percentiles of the Colorado intrauterine growth charts), except for one large for dates infant with RDS and another control of the same age, both offspring of diabetic mothers. The diagnosis of RDS was based on typical $x$-ray changes, course of disease, and physical findings $(9,12)$.

The specimens of lung effluent were obtained in the same way as described earlier (15). In addition, stomach aspirates were collected within $1 \mathrm{hr}$ after birth. In six cases stomach and retropharyngeal aspirates were obtained simultaneously. These samples essentially were identical in phospholipid composition. However, the pharyngeal specimens were smaller than those from the stomachs and sometimes were too small to be analyzed 
for phospholipids. Lung effluent after the first neonatal hour was obtained by retropharyngeal suctioning or, when indicated, by direct laringoscopy. Tracheal aspirates were collected from intubated patients during routine suctioning. When the sample was small visually, two or more successive aspirates were combined.

The aspirates collected in sterile tubes either were extracted immediately for lipids or stored at $-20^{\circ}$. Microscopic blood was separated as follows. The sample was suspended in isotonic saline using a Potter-Elvehjem homogenizer and layered on 1.64 $\mathrm{M} \mathrm{NaBr}$, followed by ultracentrifugation at $27,000 \mathrm{rpm}$ for $1 \mathrm{hr}$ using an SW 27 rotor. The interphase between the two layers was collected as surfactant; erythrocytes sedimented in the pellet and some of the serum phospholipids remained above the interphase. In some infants who died the alveolar wash was obtained by endobronchial lavage with isotonic saline (15). When separately indicated, the surfactant was purified further using density gradient and differential centrifugation as described previously (22).

Lipids were extracted as follows. The lung effluent was collected and isotonic saline was added to a volume of $5 \mathrm{ml}$. The mixture was homogenized with a few strokes from a PotterElvehjem homogenizer. Five milliliters of methanol and $10 \mathrm{ml}$ chloroform were added and the resulting mixture was stirred for $10 \mathrm{~min}$. Individual phospholipids were isolated with two-dimensional thin layer chromatography using silica gel $\mathrm{H}$ (E. Merck): first dimension: tetrahydrofuran-methylal-methanol-2 $\mathrm{M}$ $\mathrm{NH}_{4} \mathrm{OH}(10 / 8 / 2 / 1.1, \mathrm{v} / \mathrm{v})$; second dimension: chloroform-methanol $\mathrm{H}_{2} \mathrm{O}(65 / 25 / 4, v / v)$. If the amount of lipid extract were sufficient another chromatogram was developed using the following solvents: first dimension: chloroform-methanol-58\% $\mathrm{NH}_{4} \mathrm{OH}-\mathrm{H}_{2} \mathrm{O}(130 / 60 / 5 / 4, \mathrm{v} / \mathrm{v})$; second dimension: chloroformmethanol-acetic acid- $\mathrm{H}_{2} \mathrm{O}(160 / 50 / 12 / 4, \mathrm{v} / \mathrm{v})$. The individual spots were quantified by measuring the phosphorus content (20).

PG was isolated from pooled lung effluent of the newborn and identified using the chemical methods previously described (20). The present chromatography method clearly separates PG from other compounds. In addition, the presumptive PG spot in individual runs immediately gave a blue periodate-Schiff reaction (34). This feature, characteristic for an $\alpha$-substituted glycerol, differentiates it from other phospholipids.

Disaturated lecithins were isolated as described earlier (24). Fatty acids esterified in $\alpha$ and $\beta$ positions of lecithins first were separated by phospholipase $\mathrm{A}_{2}$ (pure venom from Naja naja, from Miami Serpentarium, Miami, Fla.) and then analyzed by gas liquid chromatography (16). Individual fatty acid peaks were identified using appropriate standards, and quantified according to the weight percentage. Before analysis the lecithin samples were divided into two parts: one for the analysis of $\alpha$ and $\beta$ fatty acids and the other for total fatty acids. Measurements were acceptable only when the sum of the fatty acids esterified in $\alpha$ and $\beta$ positions closely resembled the fatty acids from total lecithin.

The "neutral lipid" fraction was isolated on DEAE-cellulose columns as described by Gluck et al. (14). Individual lipids were isolated on thin layer plates of silica gel $\mathrm{H}$ containing $5 \%$ ammonium sulfate, as previously described (22).

Surface activity was measured using a modified Wilhelmy balance (22).

\section{RESULTS}

\section{COMPARISON OF LUNG EFFLUENT BETWEEN RDS AND CONTROL SUBJECTS}

The aim of this study was to obtain specimens of lung effluent at birth, day 1 , day 2 , and, if the patients were intubated, just before extubation. We often failed to get the first sample since more than half of the patients originated from surrounding hospitals, although in some cases four to six specimens were obtained during the first 2 neonatal days. In healthy control subjects or in infants with conditions other than RDS, lung effluent was collected within a few hours after birth.

Figure 1 shows the content of PG in lung effluent obtained between 1 and $48 \mathrm{hr}$ after birth. One case represents the mean of one to six measurements. PG always was absent in RDS. In control subjects of more than 28 weeks it always was present.

In control infants of 28 weeks or less PG was practically absent in five cases. One of those patients did not have typical RDS; however, he developed cardiac failure with patent ductus arteriosus and radiologic changes similar to RDS 1 week after birth PG first appeared gradually during recovery. This resembled chronic pulmonary insufficiency observed in very premature babies (26). Two other cases also required mechanical ventilation and supplemental oxygen, but did not have x-ray changes typical for RDS. The last two cases had apnea that responded well to theophylline. For those who survived PG appeared at $27-$ 29 weeks of gestation.

The percentage of PI measured in the same samples as in Figure 1 are shown in Figure 2. In infants with RDS of less than 30 weeks of gestation, PI was significantly lower than in control subjects of the same gestational age. In more mature age groups this difference was not evident; in fact, PI tended to be higher in infants with RDS than in control infants. However, beyond 30 weeks of gestation, individual variation was considerable, and therefore PI, in contrast to PG, seemed to be of value in differentiating between control subjects and those with RDS only in the group of youngest infants by gestation.

Tables 1 and 2 show the total phospholipid pattern and the fatty acids esterified to lecithin from the same samples as in the

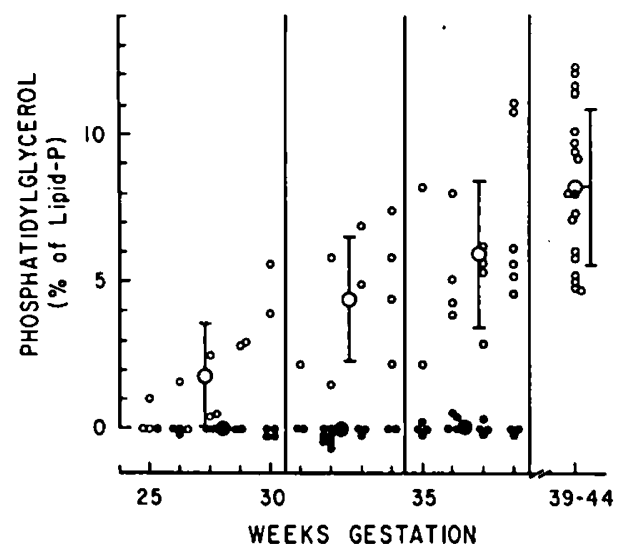

Fig. 1. The content of phosphatidylglycerol in lung effluent of newborns from $1-48 \mathrm{hr}$ after birth. Samples during recovery from respiratory distress syndrome (RDS) are not included. O, controls; $\bullet$, cases of RDS. Each point represents the mean of from one to six measurements from one individual.

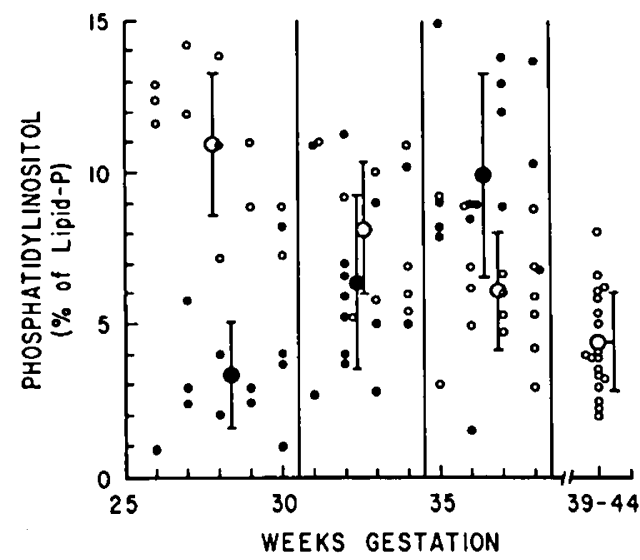

Fig. 2. The content of phosphatidylinositol in lung effluent of the newborn. Other details as described for Figure 1. 
Table 1. Molar contents of phospholipids as percentage of total lipid-phosphorus isolated from lung effluent from $1-48$ hr after birth ${ }^{1}$

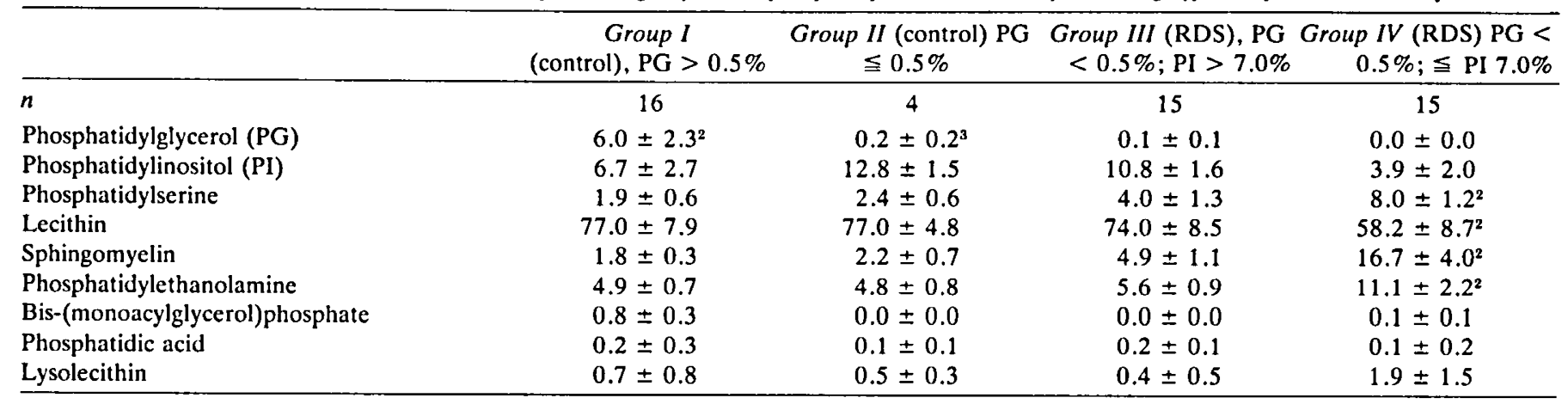

' Only those cases were included when the material was sufficient for thin layer chromatography with the two solvent systems (see "Methods"). RDS: respiratory distress syndrome.

${ }^{2}$ Significant $(P<0.001)$ difference as compared to the other three groups.

${ }^{3}$ Mean \pm SD.

Table 2. Percentage composition of $\beta$ fatty acids of lecithins isolated from lung effluent from $1-48 \mathrm{hr}$ after birth

\begin{tabular}{|c|c|c|c|c|}
\hline & $\begin{array}{c}\text { Group I (control), PG > } \\
0.5 \%^{1} \\
\end{array}$ & $\begin{array}{c}\text { Group II (control), PG } \leqq \\
0.5 \% \\
\end{array}$ & $\begin{array}{c}\text { Group III (RDS), PI > } \\
7.0 \% \\
\end{array}$ & $\begin{array}{c}\text { Group IV (RDS), PI } \leqq \\
7.0 \% \\
\end{array}$ \\
\hline$n$ & 7 & 6 & 13 & 4 \\
\hline $14: 0$ & $5.8 \pm 1.4^{2}$ & $6.0 \pm 1.7$ & $6.2 \pm 1.9$ & $4.1 \pm 1.1$ \\
\hline $16: 0$ & $54.8 \pm 6.9$ & $54.0 \pm 7.4$ & $44.9 \pm 6.0$ & $32.0 \pm 5.8^{3}$ \\
\hline $16: 1$ & $11.6 \pm 5.4$ & $8.4 \pm 5.5$ & $13.4 \pm 4.1$ & $7.8 \pm 4.3$ \\
\hline 18:0 & $3.4 \pm 1.8$ & $3.6 \pm 4.3$ & $7.0 \pm 4.0$ & $3.3 \pm 1.9$ \\
\hline $18: 1$ & $15.3 \pm 2.0$ & $17.6 \pm 4.1$ & $19.6 \pm 7.2$ & $22.9 \pm 5.0$ \\
\hline $18: 2$ & $7.1 \pm 3.1$ & $8.5 \pm 2.8$ & $6.9 \pm 2.5$ & $7.4 \pm 2.1$ \\
\hline $18: 3+20: 1$ & $1.2 \pm 1.0$ & $1.0 \pm 1.1$ & $1.0 \pm 1.0$ & $2.9 \pm 1.6$ \\
\hline $22: 0$ & $0.8 \pm 0.8$ & $0.9 \pm 0.5$ & $1.1 \pm 0.8$ & $1.1 \pm 1.2$ \\
\hline Unknown & $0.0 \pm 0.0$ & $0.0 \pm 0.0$ & $0.0 \pm 0.0$ & $8.5 \pm 3.0^{3}$ \\
\hline$\%$ Saturated & $64.8 \pm 7.4$ & $64.5 \pm 7.4$ & $59.1 \pm 8.7$ & $40.5 \pm 6.7^{3}$ \\
\hline
\end{tabular}

${ }^{1}$ The amount of phosphatidylglycerol (PG) and phosphatidylinositol (PI) as a percentage of total lipid phosphorus.

${ }^{2}$ Mean \pm SD.

${ }^{3}$ Significant $(P<0.005)$ difference as compared to the other three groups.

previous figures. Table 1 includes only those cases that were assayed for phospholipids using two different solvent systems (see "Methods"). Four groups were included. The first represents those cases who did not have RDS and whose PG exceeded $0.5 \%$ of phospholipid-P. The second group includes those control subjects who had $0.5 \%$ or less PG. The third represents cases of RDS where PI exceeded $7 \%$ of phospholipid-P, and the fourth RDS with $7 \%$ or less PI. These limits for PI content are artificial; however, in amniotic fluid PI exceeding $7 \%$ is likely to be associated with a mature $\mathrm{L} / \mathrm{S}$ ratio $(23)$. Therefore $7 \%$ of $\mathrm{PI}$ was chosen as a limit.

The contents of sphingomyelin and phosphatidylserine were higher in infants with RDS than in the control subjects $(P<$ $0.001)$. When PI was low in those infants with RDS, their lecithin contents also were significantly lower than in the control subjects (Table 1). This suggests the paucity of surfactant. The fatty acids esterified to the $\alpha$ position of lecithin did not differ significantly among the four groups ( $c f$. Reference 15$)$. The fatty acids in the $\beta$ position are shown in Table 2 . They were similar in the first three groups. However, a significantly lower percentage of saturated fatty acids was present in the fourth group comprising cases of RDS with low PI. Palmitate always was the most prominent saturated fatty acid. Representative chromatograms are shown in Figure 3.

We further studied whether the composition of acidic phospholipids in tracheal effluent at birth could reflect an infant's future outcome. Figure 4 shows the content of acidic phospholipids as a function of age after birth. The absence of PG at birth did not necessarily indicate that RDS was inevitable, since PG may appear during the first neonatal hour. On the other hand, in
RDS, surfactant frequently was present at birth, as indicated by high PI levels. However, during the subsequent hours, PG failed to appear. The underlying control mechanisms of this induction and its failure need further study.

An attempt was made to correlate the levels of PI at birth and the severity of RDS. The severity of the disease possibly was milder when surfactant was found in the airways at birth (PI greater than $7 \%$ of phospholipid-P). However, all degrees of severity were observed even when surfactant was present (data not shown).

Figure 5 shows the content of the acidic phospholipids, L/S ratio, and percentages of disaturated lecithins in lung effluent during the course of one representative infant with RDS who had surfactant at birth. L/S ratios, percentage of disaturated lecithins, and PI content remained high but PG gradually appeared first during recovery. PG frequently remained significantly lower than in the control subjects, even after the disappearance of $x$-ray changes typical to RDS.

The total amount of phospholipids recovered from lung effluent seemed to depend mainly on the quantity of surfactant produced, but there was considerable individual variation. At the height of RDS little phospholipid could be recovered during a single suctioning (days 1-2: $0.8 \pm 0.4 \mu \mathrm{mol}, n=4$ ) and during recovery from RDS (days $4-8: 3.9 \pm 2.0 \mu \mathrm{mol}, n=11$ ). This suggests that the amount of surfactant in the airways correlates with the degree of atelectasis.

\section{STUDIES ON ISOLATED SURFACTANT COMPLEX}

In 20 cases the surfactant complex was isolated using a density gradient and differential centrifugation. When the aspirates from 
infants with RDS contained less than $7 \%$ of PI (three cases), $30 \%$ or less of material was recovered as surfactant complex. The possible source of lung effluent in these cases include serum and membranes from destroyed epithelial lining. However, lung
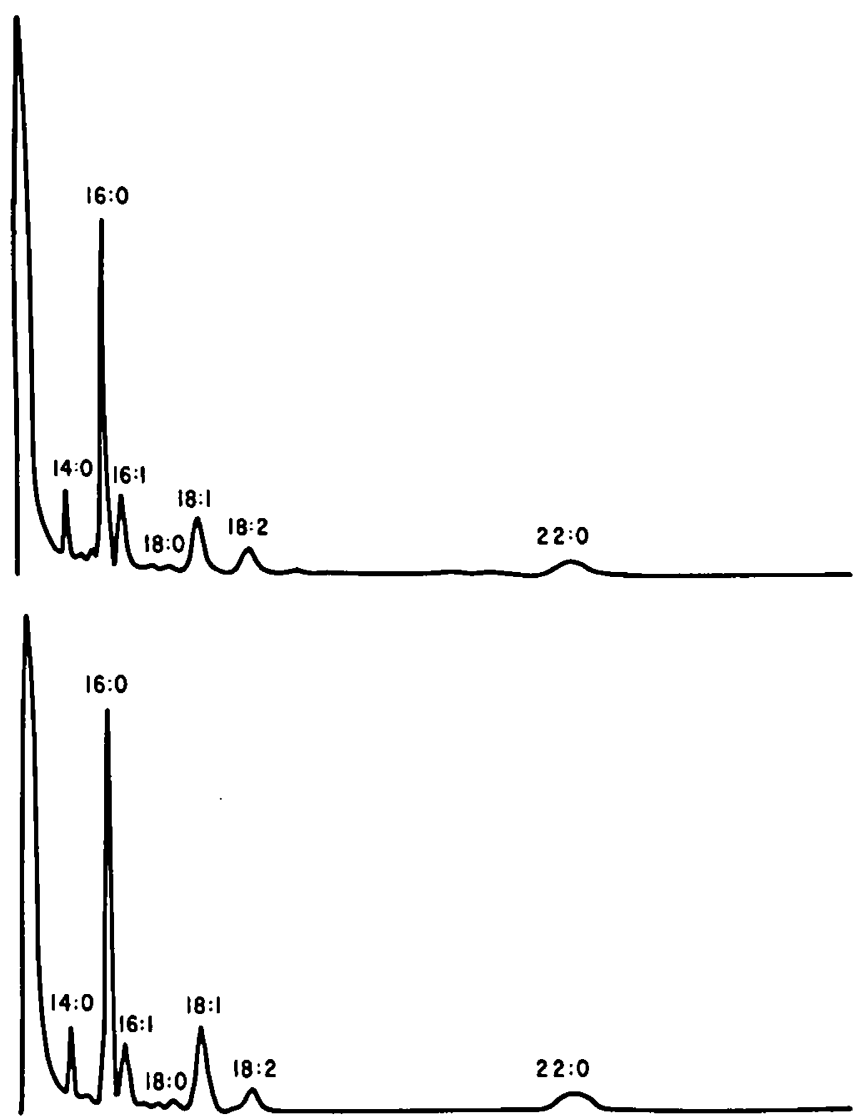

Fig. 3. The fatty acids in $\beta$ carbons of surfactant lecithins. Above: alveolar lavage from 2-day-old baby (birth weight $1200 \mathrm{~g}, 29$ weeks of gestation) with severe respiratory distress syndrome (RDS) who died from intraventricular hemorrhage. Below: tracheal aspirate from 2-dayold baby (birth weight $1020 \mathrm{~g}, 28$ weeks of gestation) with no RDS. Phosphatidylglycerol content of purified surfactant was $4.5 \%$ of lipidphosphorus. Tracings of gas chromatograph are shown.
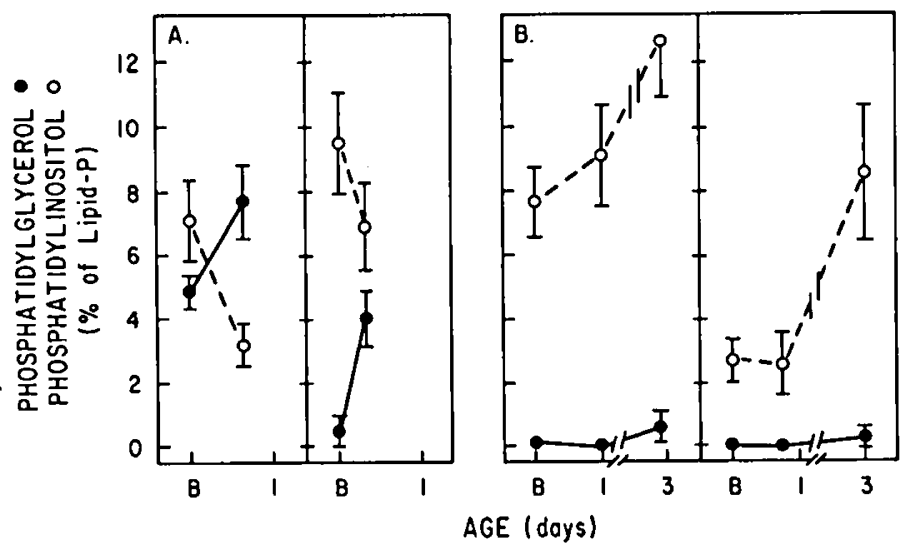

Fig. 4. The content of acidic phospholipids following birth. $A$ : no respiratory distress syndrome (RDS). Left: phosphatidyglycerol (PG) greater than $0.8 \%$ of lipid-phosphorus at birth. Right: PG equal to or less than $0.8 \%$ of lipid-phosphorus at birth. B: RDS. Lefi: phosphatidytinositol (PI) greater than 7\% of lipid-phosphorus at birth. Right: PI equal to or less than $7 \%$ of lipid-phosphorus at birth. PG both right and left less than $0.8 \%$ of lipid-phosphorus. The results are the mean $\pm S D$ of two to eight cases. effluent with high PI from babies with RDS contained $50 \%$ or more material characteristic of surfactant in density. The following evidence further indicated that this material was surfactant. (1) The fatty acid pattern of lecithin was virtually identical with human surfactant lecithin. (2) The lecithin fraction was surface active; i.e., it decreased the surface tension below 10 dynes $/ \mathrm{cm}$ on the surface balance. (3) The phospholipid composition was similar to the control group II in Table 1 (PI: $13.6 \pm 2.8 \%$ of phospholipid-P).

To further study the possible differences between the surfactants with and without PG "neutral lipids" were assayed in infants with RDS (PG: $0.0 \pm 0.0 \%$ phospholipid-P, $n=8$ ) and in control subjects (PG: $6.8 \pm 1.8 \%$ phospholipid-P, $n=5$ ). No significant difference between these two groups was found; cholesterol (RDS: $0.09 \pm 0.02 \mu \mathrm{g} / \mu \mathrm{g}$ phospholipids, $47.8 \%$ of "neutral lipids"; control: $0.09 \pm 0.01 \mu \mathrm{g} / \mu \mathrm{g}$ phospholipid $45.8 \%$ of "neutral lipids") was the most prominent component in each case.

The physicochemical characteristics of lung effluent were studied on a modified Wilhelmy balance using purified surfactant. In RDS infants the minimum surface tensions were significantly higher $(17.2 \pm 1.9$ dynes $/ \mathrm{cm})$ than in the control subjects (12.1 \pm 2.0 dynes $/ \mathrm{cm})$. Representative measurements are shown in Figure 6. In RDS the surface tension of purified surfactant failed to decrease much below 20 dynes $/ \mathrm{cm}$, whereas the controls showed a sharp decrease in surface tension when the surface was compressed.

\section{LUNG EFFLUENT IN CONDITIONS OTHER THAN RDS}

Table 3 indicates the distribution of acidic phospholipids in respiratory disturbances of the newborn other than RDS. In subjects with pneumonia, levels of PG and PI were similar to those of control subjects. However, in the only case of septicemia due to $\beta$-hemolytic Streptococcus type B studied, there was low PG in four consecutive samples, suggesting either possible arrest in maturation or else significant destruction of surfactant synthesizing cells. Also, the five cases of RDS type II (retained lung fluid syndrome) had low PG and high PI. After recovery the acidic phospholipids were normal (two cases).

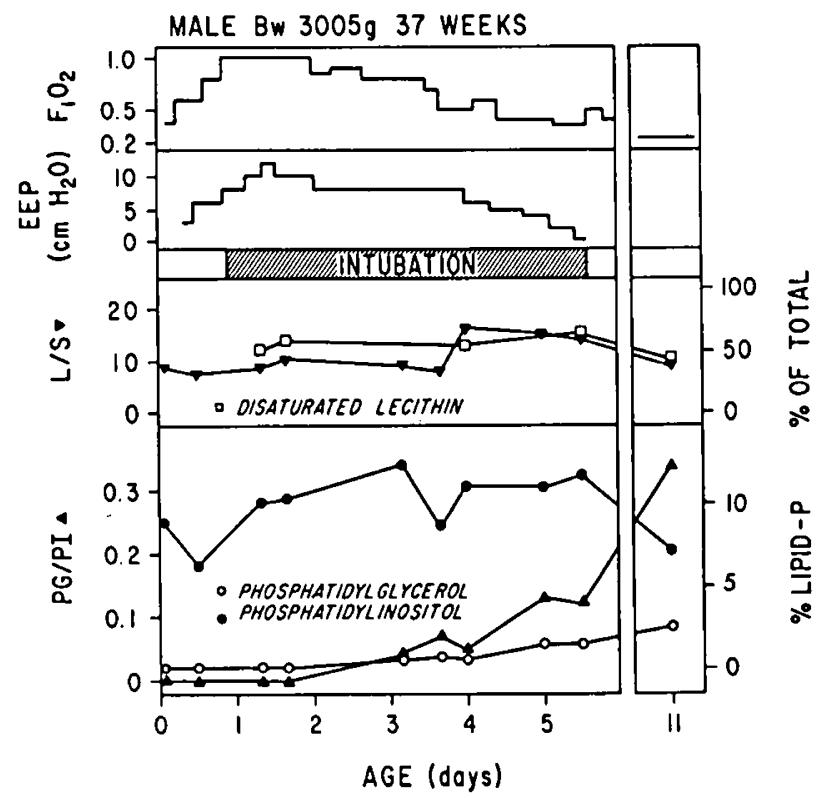

Fig. 5. Changes in lung effluent of an infant with respiratory distress syndrome (RDS). Inspiratory oxygen contents $\left(F_{1} O_{2}\right)$ and end-expiratory pressures $(E E P)$; lecithin to sphingomyelin $(L / S)$ ratios on total lipid extracts: percentage of disaturated lecithin of total lecithin; phosphatidylinositol (PI) and phosphatidylglycerol (PG) (\% of lipid-P) and PG/PI ratio shown. 
In six cases of postnatal and perinatal asphyxia, the acidic phospholipids were apparently normal; in one case PG was even abnormally high. Only one of the infants was monitored at birth, and even then had prominent PG. The two premature babies with severe perinatal asphyxia (cases 7 and 8 with 1 min Apgar scores of 1 and 3, respectively) did not develop PG after birth and RDS ensued.

\section{DISCUSSION}

\section{RDS - ABSENT PHOSPHATIDYLGLYCEROL}

The virtual absence of $P G$ from lung effluent in the newborn with RDS was demonstrated in this study. In striking contrast

Table 3. Content of acidic phospholipids in lung effluent of neonates with various respiratory disturbances ${ }^{1}$

\begin{tabular}{|c|c|c|c|}
\hline \multirow[b]{2}{*}{ Diagnosis } & \multirow{2}{*}{$\begin{array}{c}\text { Gesta- } \\
\text { tional } \\
\text { age, } \\
\text { weeks }\end{array}$} & \multicolumn{2}{|c|}{$\%$ of phospholipid-P } \\
\hline & & PG & PI \\
\hline \multicolumn{4}{|l|}{ Pneumonia } \\
\hline 1: Cytomegalovirus & 30 & $9.4(4.8)$ & $3.5(8.1)$ \\
\hline 2: Candida albicans & 29 & $2.9(2.0)$ & $8.9(9.9)$ \\
\hline 3: Streptococcus group B & 38 & $1.0(7.2)$ & $10.3(5.7)$ \\
\hline 4: Meconium aspiration & 40 & $9.8(9.0)$ & $2.2(3.8)$ \\
\hline 5: Meconium aspiration & 40 & $7.9(9.0)$ & $4.5(3.8)$ \\
\hline \multicolumn{4}{|l|}{ RDS type II } \\
\hline 1 & 37 & $1.3(5.0)$ & $9.3(5.7)$ \\
\hline 2 & 34 & $1.4(4.9)$ & $8.9(7.3)$ \\
\hline 3 & 26 & $0.3(0.8)$ & $11.5(12.3)$ \\
\hline 4 & 37 & $1.6(5.0)$ & $8.9(5.7)$ \\
\hline 5 & 39 & $1.7(6.1)$ & $10.0(5.4)$ \\
\hline \multicolumn{4}{|l|}{ Asphyxia } \\
\hline 1: Congenital heart disease & 40 & $9.8(9.0)$ & $2.9(3.8)$ \\
\hline 2: Congenital heart disease & 42 & $10.4(9.6)$ & $3.9(3.5)$ \\
\hline 3: Congenital heart disease & 40 & $5.1(9.0)$ & $4.7(3.8)$ \\
\hline 4: Perinatal & 38 & $3.4(7.2)$ & $9.9(5.7)$ \\
\hline 5: Perinatal & 38 & $5.2(7.2)$ & $5.9(5.7)$ \\
\hline 6: Perinatal & 42 & $14.8(9.6)$ & $4.2(3.5)$ \\
\hline 7: Perinatal + RDS & 36 & $0.6(5.3)$ & $10.0(6.7)$ \\
\hline 8: Perinatal + RDS & 35 & $0.2(5.2)$ & $14.9(6.1)$ \\
\hline
\end{tabular}

1 Numbers in parentheses indicate the content of acidic phospholipid in control subjects of the same gestational age. RDS: respiratory distress syndrome; PG: phosphatidylglycerol; PI: phosphatidylinositol. this phospholipid was always present in full term babies: healthy newborns, newborns with pneumonia, and asphyxiated newborns. The earlier investigations of Gluck and associates (15) suggested that a precursor of lecithin, PDME, was absent in RDS, indicating possible absence of inhibition of synthesis of lecithin by the methylation pathway. It was suggested that when the methylation pathway was absent, the choline incorporation pathway did not provide sufficient surfactant in the premature baby and RDS ensued.

The identification of PDME in dog lung was first reported by Morgan et al. (29) and presumptive verification made by Gluck et al. (16). However, recent evidence $(22,31,33)$ indicates that substantial amounts of PG and not PDME are present in adult and newborn surfactant. On the other hand, present data on the distribution of PG essentially confirm the earlier primary findings of Gluck and associates, although the interpretation of the results is different.

\section{RDS - SURFACTANT NOT NECESSARILY ABSENT}

The present findings agree with earlier findings on the deficiency of surfactant in the airways of most newborns with RDS $(3,15)$. In these cases surfactant could be found in the airways only after one or several days of life, its appearance heralding the recovery phase. However, in about $40 \%$ of the present patients sampled, surfactant complex was found in tracheal effluent even in the early stages of the disease. Of these, $72 \%$ were of gestational age of 35 weeks or more, three were infants of diabetic mothers, and two were born after severe perinatal asphyxia. The clinical hallmarks and the $x$-ray findings essentially were indistinguishable from RDS where surfactant could not be found. The findings of surfactant in RDS agree with that of Lauweryns (27) and Balis et al. (4), who sometimes detected impressive numbers of lamellar bodies apparently representing intracellular collections of surfactant in patients with RDS.

The present study focuses on the qualitative aspects of surfactant; this could be equally as important as the quantity of surfactant in stabilizing the alveoli. In normal pregnancy PI that presumably is derived from the lung (22) increases in amniotic fluid after the 30 th gestational week. PG first appears after the 35 th week (23). Birth may affect the normally gradual development of the acidic phospholipids in the fetus: in some healthy prematures PG appeared rapidly in lung effluent within the first neonatal hour. However, in RDS it gradually developed only during recovery. The failure of induction of $P G$ with prominent PI at birth is remarkable and suggests that some regulatory factor(s) may be defective.

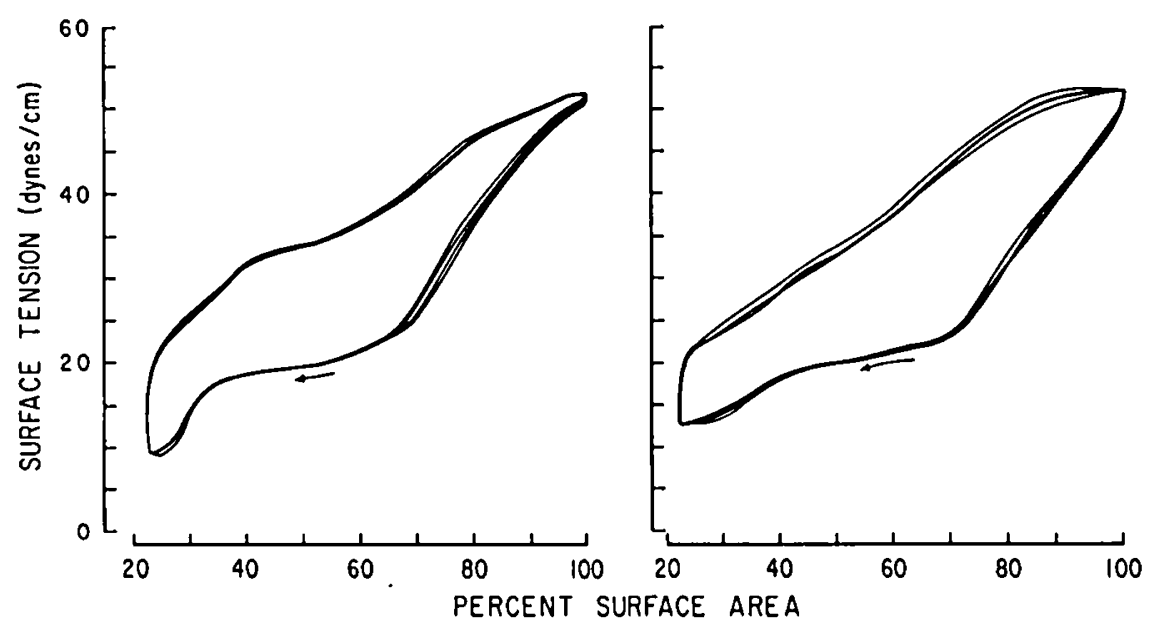

Fig. 6. The recordings of surface tension-area relationship on a modified Wilhelmy balance of purified surfactant from a 37-week-old baby at birth (lefi) and from an infant of a diabetic mother with RDS (birth weight $3500 \mathrm{~g}$ ), 36 weeks of gestation (right). The compression of the surface is indicated by the arrows. The amount of phospholipid applied to the surface was $30 \mathrm{nmol}$, representing $10 \AA^{2} /$ molecule during maximal compression of the surface. 
In RDS type II (retained lung fluid syndrome) the contents of PG were low and PI prominent. During recovery the acidic phospholipids normalized. This suggests that the retained lung fluid syndrome may be a mild form of RDS with somewhat immature surfactant but sufficient in function to prevent atelectasis.

\section{POSSIBLE SIGNIFICANCE OF ACIDIC SURFACTANT PHOSPHOLIPIDS}

The present evidence thus far suggests that PG improves the quality of surfactant that may be critical in stabilizing the alveoli. The tubular airways of the bird lung (22) as well as the alveolar ducts of the very premature lung contain little, if any, PG but prominent PI, suggesting that the requirements for surfactant may vary on the basis of the structure of the terminal airways. Moreover, according to the LaPlace formula, the pressure (P) tending to collapse the curved surface is dependent on the shape as follows

$$
\mathrm{P}=\mu\left(\frac{1}{\mathrm{R}}+\frac{1}{\mathrm{r}}\right)
$$

where $\mu=$ surface tension; $\mathrm{R}$ and $\mathrm{r}=$ the principal radii of the curvature. Thus, in a sphere (alveolus), the collapsing pressure $\left(\mathrm{P}_{\mathrm{a}}=2 \mu / \mathrm{r}\right)$ is twice as great as in a cylinder (tubular airway) of the same surface tension and radius $\left(P_{1}=\mu / r\right)$. Further studies are needed to establish dependence between the structure of the terminal airways and the composition and physicochemical characteristics of surfactant.

The present findings agree with the hypothesis that a defect in surfactant is a primary factor in the pathogenesis of RDS (11, $13,15)$. The amount of surfactant in the airways at the height of the disease is always diminished. However, in some cases, such as most of those beyond 35 weeks of gestation, the qualitative rather than the quantitative aspects of surfactant may be the major cause of the disease.

The rate of surfactant production and its release into the airways increase during perinatal development $(11,13,32)$. At the same time the individual components characteristically change in concentration $(21,22)$. The complexities of surfactant ontogeny imply multiple control mechanisms. Studies in this area may further refine the diagnosis as well as open new ways of prevention and therapy.

\section{CONCLUSION}

In RDS phosphatidylglycerol (PG) was always absent in lung effluent, whereas lung effluent from healthy newborns and those with pneumonia or cardiovascular disease contained this phospholipid. However, in some cases of RDS, even in the early stages of the disease, surface-active lecithin was present in the airways. The measurements on a surface balance suggest that surfactant with PG as compared to that without PG has better physicochemical characteristics to guarantee alveolar stability.

PG as a component of the surfactant complex serves as a biochemical marker: in its absence there is a significant risk for developmental lung disease; the presence of PG suggests biochemical maturity of the surfactant.

\section{REFERENCES AND NOTES}

1. Adams, F. H., Fujiwara, T., Emmanoulides, G., and Scudder, A.: Surface properties and lipids from lungs of infants with hyaline membrane disease. $J$. Pediat., 66: 357 (1965).

2. Auld, P. A. M., Nelson, N. M., Cherry, R. B., Rudolph, A. J., and Smith, C. A.: Measurement of thoracic gas volume in the newborn infant. J. Clin. Invest., 42: 476 (1963).

3. Avery, M. E., and Mead, J.: Surface properties in relation to atelectasis and hyaline membrane disease. Amer. J. Dis. Child., 97: 517 (1959).

4. Balis, J. V., Delivoria, M., and Correro, P. E.: Maturation of postnatal human lung and the idiopathic respiratory distress syndrome. Lab. Invest., 15: 530 (1966).

5. Brumley, G. W., Hodson, W. A., and Avery, M. E.: Lung phospholipids and surface tension correlations in infants with and without hyaline membrane disease and in adults. Pediatrics, 40:13,13 (1967).

6. Chernick, V., and Vidyasagar, D.: Continuous negative chest wall pressure in hyaline membrane disease: One year experience. Pediatrics, 49: 753 (1972).

7. DeLemos, R. A., Shermeta, D. W., Knelson, J. H., Kotas, R., and Avery, M. E.: Acceleration of appearance of pulmonary surfactant in the fetal lamb by administration of corticosteroids. Amer. Rev. Resp. Dis., I02: 459 (1970).

8. Epstein, M. F, and Farrell, P. M.: Choline incorporation pathway-Primary mechanism for de novo lecithin synthesis in fetal primate lung. Pediat. Res., 9: 658 (1975).

9. Farrell, P. M., and Avery, M. E.: Hyaline membrane disease. Amer. Rev. Resp. Dis., 111: 657 (1975).

10. Feldman, B. H., Mannino, F., Delue, N. A., Heldt, G. P., Wimmer, J. E. Fletcher, M. A., and Gluck, L.: Mechanical ventilation in respiratory distress syndrome: Early intervention with prolonged inspiratory time. (Submitted for publication.)

11. Gluck, L., and Kulovich, M. V.: Lecithin/sphingomyelin ratios in amniotic fluid in normal and abnormal pregnancy. Amer. J. Obstet. Gynecol., 115: 539 (1973).

12. Gluck, L., Kulovich, M. V., and Borer, R. C., Jr.: Estimates of fetal lung maturity. Clin. Perinatol., 1: 125 (1974).

13. Gluck, L., Kulovich, M. V., Borer, R. C., Jr., Brenner, P. H., Anderson, G. G., and Spellacy, W. N.: Diagnosis of the respiratory distress syndrome by amniocentesis. Amer. J. Obstet. Gynecol., 109: 440 (1971).

14. Gluck, L., Kulovich, M. V., and Brody, S. J.: Rapid quantitative measurement of lung tissue phospholipids. J. Lipid Res., 7: 570 (1966).

15. Gluck, L., Kulovich, M. V., Eidelman, A. I., Cordero, L., and Khazin, A. F. Biochemical development of surface activity in mammalian lung. IV. Pulmonary lecithin synthesis in the human fetus and newborn and etiology of the respiratory distress syndrome. Pediat. Res., 6: 81 (1972).

16. Gluck, L., Landowne, R. A., and Kulovich, M. V.: Biochemical development of surface activity in mammalian lung. III. Structural changes in lung lecithin during development of the rabbit fetus and the newborn. Pediat. Res., 4: 352 (1970).

17. Gluck, L., Scribney, M., and Kulovich, M. V.: The biochemical development of surface activity in mammalian lung. II. The biosynthesis of phospholipids in the lung of the developing rabbit fetus and newborn. Pediat. Res., 1: 247 (1967).

18. Gregory, G. A., Kitterman, J. A., Phibbs, R. H., Tooley, W. H., and Hamilton, W. K.: Treatment of the idiopathic respiratory distress syndrome with continuous positive airway pressure. N. Engl. J. Med., 284: 1333 (1971).

19. Grumwald, P., Johnson, R. P., Hustead, R. F., and Clements, J. A.: Correlation of mechanical properties of infant's lungs with surface activity of extracts. Proc. Soc. Exp. Biol. Med., 109: 369 (1962).

20. Hallman, M., and Gluck, L.: Phosphatidylglycerol in lung surfactant. I. Synthesis in rat lung microsomes. Biochem. Biophys. Res. Commun., 60: 1 (1974).

21. Hallman, M., and Gluck, L.: The biosynthesis of phosphatidylglycerol in the lung of the developing rabbit. Fed. Proc., 34: 274 (1975).

22. Hallman, M., and Gluck, L.: Phosphatidylglycerol in lung surfactant. III. Possible modifier of surfactant function. J. Lipid Res., 257 (1976).

23. Hallman, M., Kulovich, M. V., Kirkpatrick, E., Sugarman, R. G., and Gluck, L.: Phosphatidylinositol (PI) and phosphatidylglycerol (PG) in amniotic fluid: Indices of lung maturity. Amer. J. Obstet. Gynecol., 125: 613 (1976)

24. Hallman, M., and Raivio, K.: Studies on the biosynthesis of disaturated lecithin of the lung: The importance of the lysolecithin pathway. Pediat. Res., 8: 874 (1974).

25. Karlberg, P., Cook, C. D., OBrien, D., Cherry, R. B., and Smith, C. A.: Studies of respiratory physiology in the newborn infant: Observations during and after respiratory distress. Acta Paediat., 43: 397 (1954).

26. Krauss, A. N., Klain, D. B., and Auld, P. A. M.: Chronic pulmonary insufficiency of prematurity (CPIP). Pediatrics, 55: 55 (1975).

27. Lauweryns, J. M.: "Hyaline membrane disease" in newborn infants: Macroscopic, radiographic and light and electron microscopic studies. Human Pathol., 1: 175 (1970).

28. Liggins, G. C., and Howie, R. N.: A controlled trial of antepartum glucocorticoid treatment for prevention of the respiratory distress syndrome in premature infants. Pediatrics, 50: 515 (1972).

29. Morgan, T. E., Finley, T. N., and Fialkow, H.: Comparison of the composition and surface activity of alveolar and whole lung lipids in the dog. Biochim. Biophys. Acta, 106: 403 (1965).

30. Motoyama, E., Orzalesi, M., Kikkawa, Y., Kaibara, M., Wu, B., Zigas, C. and Cook, C. D.: Effect of cortisol on the maturation of fetal rabbit lung. Pediatrics, 48 : 547 (1971).

31. Pfleger, R. C., and Thomas, H. G.: Beagle dog pulmonary surfactant lipids. Lipid composition of pulmonary tissue, exfoliated lining cells and surfactant. Arch. Intern. Med., 108: 253 (1971).

32. Platzker, A. C. G., Kitterman, J. A., Mescher, J., Clements, J. A., and Tooley, W. H.: Surfactant in the lung and tracheal fluid of the fetal lamb and acceleration of its appearance by dexamethasone. Pediatrics, 56: 554 (1975).

33. Rooney, S. A., Canavan, P. M., and Motoyama, E. K.: The identification of phosphatidylglycerol in the rat, rabbit, monkey and human lung. Biochim. Biophys. Acta, 360: 56 (1974).

34. Shaw, N.: The detection of lipids on thin-layer chromatograms with the periodate-Schiff reagents. Biochim. Biophys. Acta, 164: 435 (1968).

35. Weinhold, P.: Biosynthesis of phosphatidylcholine during neonatal develop- 
ment of the rat lung. J. Lipid Res., 9: 262 (1968).

36. Informed consent was obtained for all specimens obtained from patients.

37. The present address of Dr. Mikko Hallman is: Children's Hospital, University of Helsinki, Stenbackinkatu 11, 00290 Helsinki 29, Finland.

38. The present address of Dr. Bernard H. Feldman is: Medical Director, Southwestern Neonatal Intensive Care Unit, Sunrise Hospital, Las Vegas, Nev. 89109.
39. This research was supported by National Institutes of Health Grant HD 04380, SCOR HL 14169, TW-02051.

40. Requests for reprints should be addressed to: Louis Gluck, M. D., Department of Pediatrics/C-019, University of California, San Diego, La Jolla, Calif. 92093 (USA).

41. Received for publication April 30, 1976.

42. Accepted for publication October 8, 1976.
Alanine

amino acids carbohydrates glutamate newborn

postnatal period protein biosynthesis

\title{
Early Postnatal Metabolism of Amino Acids in Rat
}

\author{
THIERRY CRESTEIL (24) AND JEAN-PAUL LEROUX \\ Laboratoire de Biochimie, INSERM U.75, CHU Necker-Enfants Malades, Paris, France
}

\section{Summary}

The production of ${ }^{14} \mathrm{CO}_{2}$ from glutamate and alanine studied in the newborn rat after injection of labeled precursors was intense and revealed an important catabolism from $130 \mathrm{nmol} / \mathrm{hr}$ in a 0-hr neonate to $2000 \mathrm{nmol} / \mathrm{hr}$ in 3-week-old rat. The degradative metabolism of leucine was low at birth $(10 \mathrm{nmol} / \mathrm{hr})$, but increased up to $500 \mathrm{nmol} / \mathrm{hr}$ in 3-week-old rat. Phenylalanine was practically not metabolized into $\mathrm{CO}_{2}$, but its incorporation into protein was higher than leucine, alanine, and glutamate incorporation. Glucose is the major fuel utilized by newborn rat for its energy supply $(300-560 \mathrm{nmol} / \mathrm{hr})$. However, the relative part of amino acids in energy production is important in the early neonate ( 0 and $1 \mathrm{hr}$ after birth).

\section{Speculation}

In the early newborn rat, the important oxidative metabolism of amino acids (before phosphoenolpyruvate carboxykinase (PEPCK) biosynthesis and gluconeogenesis activation) indicates that they may be directly utilized for energy requirements. This amino acid oxidation could be considered as a transient energetic pathway in species showing a delayed development of PEPCK activity (e.g., the human neonate).

In the fetus, the energy supply is provided by the mother through the placental barrier. Amino acids and glucose are the two major nutrients which cross the rat placenta: freely for glucose (18) or by an active transport mechanism for amino acids (2). Maternal glucose appears to be the major oxidative fuel for the fetus in utero (1), but it is also utilized during the last days of gestation for the constitution of endogenous stores: hepatic glycogen and triglycerides in brown adipose tissue. Amino acids are essentially used for macromolecule synthesis.

However, metabolic measurements in fetal lamb indicate that amino acids are also utilized for energy supply. The fetal urea production (a reflection of amino acid catabolism) and its excretion to the maternal circulation support this view (9).
In the first hours following birth, as during starvation, a transient hypoglycemia is observed; carbohydrate stores are then utilized for energy requirements whereas endogenous triglycerides assure thermogenesis in brown adipose tissue. An active gluconeogenesis from amino acids appears after some hours and maintains constant the plasma glucose concentration.

In the present study, the respective participations of amino acids in oxidative processes (directly or after conversion into carbohydrates) and in protein biosynthesis were investigated in vivo during the early postnatal period.

\section{MATERIALS AND METHODS}

Newborn rats (Sprague-Dawley strain) were obtained by cesarian section on the 21.5th day of gestation under light ether anesthesia. Metabolic measurements have been performed previously on control animals delivered without anesthesia; no significant differences in the ${ }^{14} \mathrm{CO}_{2}$ production were noted and the values were pooled. Labeled precursor $(0.5$ or $1 \mu \mathrm{Ci})$ was injected intraperitoneally and the respiratory ${ }^{14} \mathrm{CO}_{2}$ was monitored for $1 \mathrm{hr}$ starting immediately after injection. The relative concentrations of precursors were related to the plasma concentrations given for newborn rat in the literature. Tracer amounts of precursors were injected in a 50- $\mu$ l volume for newborn animals or $500 \mu \mathrm{l}$ for 3 -week-old rat: $\mathrm{L}-\left[U-{ }^{14} \mathrm{C}\right]$ alanine, $3 \mathrm{mM}$; $\mathrm{L}-\left[U-{ }^{14} \mathrm{C}\right]$ glutamate, $2 \mathrm{mM} ; \mathrm{L}-\left[U-{ }^{14} \mathrm{C}\right]$ leucine, $1 \mathrm{mM} ; \mathrm{L}-[U-$ $\left.{ }^{14} \mathrm{C}\right]$ phenylalanine, $0.5 \mathrm{mM}$; D- $\left[U-{ }^{14} \mathrm{C}\right]$ glucose, $40 \mathrm{mM}$.

After injection, animals were placed in a sealed dessicator (or flask) kept at $37^{\circ}$ into which humidified air was flowed at constant pressure. $\mathrm{CO}_{2}$ was collected in $\mathrm{NaOH}(10 \mathrm{~N})$ and converted into $\mathrm{BaCO}_{3}$ for radioassay with Unisolve as scintillator.

At the end of incubation, blood was collected, tissues were quickly excised, weighed, and homogenized in 5\% cold trichloroacetic acid. After centrifugation proteins were dissolved in 3 $\mathrm{ml} 88 \%$ formic acid to which $0.6 \mathrm{ml} 30 \%$ hydrogen peroxide was added. The reaction was carried on $30 \mathrm{~min}$ under magnetic stirring (13). Proteins were reprecipitated with trichloroacetic acid, added to Unisolve, and counted in a Packard scintillation spectrometer with quenching standardization. 